\title{
Study on Pulse Duration of Supercritical Carbon Dioxide Coolant Delivery on Machining Performance of AISI 1045
}

\author{
Rahim E.A. ${ }^{1}$, Amiril S. A. S., Mohid Z., Yahaya S. N. M., \\ Ibrahim M. R. and Shukuran M. F. M. \\ Precision Machining Research Center (PREMACH), Faculty of Mechanical and Manufacturing Engineering, \\ Universiti Tun Hussein Onn Malaysia, Parit Raja, 86400 Batu Pahat, Johor, Malaysia \\ 1 erween@uthm.edu.my
}

\begin{abstract}
In the new global economy, consumption has become a central issue for many fields of industries. This project work studied on the effectiveness of pulse duration of supercritical carbon dioxide $\left(\mathrm{SCCO}_{2}\right)$ in machining performance in terms of cutting temperature, cutting force and surface roughness in turning AISI 1045 medium carbon steel by uncoated cemented carbide insert. Pulse mode reduces the quantity used for cutting fluids because of the decrease of spray duration. The minimization of cutting fluids also brings economic benefits by way of saving the carbon dioxide and lubricant costs. The encouraging result includes the reduction of lubricant flow rate consumption and machining cost while maintaining significant performance, such as cutting temperature, cutting force and surface roughness. The performance of $\mathrm{SCCO}_{2}$ pulse cooling is comparable with continuous cooling with only $34 \%$ maximum increment in temperature, $3.64 \%$ increment of cutting force, $14 \%$ increment of surface roughness and $36.18 \%$ increment of tool life.
\end{abstract}

Keyword- Cryogenic coolant, Lathe, Pulse, Supercritical carbon dioxide, Sustainable machining

\section{INTRODUCTION}

In past decades, rapid development in manufacturing technology are emerging in many ways. Product quality and cost reduction are an important components in the manufacturing activities. They are some of the main key roles in obtaining the effectiveness of machining processes. Previous studies have reported that the main consideration is usually the overall machining cost, which depends most strongly in tool life [1]. The tool life and the tool wear rate are both affected by high cutting force and temperature during machining operation.

In the turning process, the temperature at the critical point is normally controlled by the cutting fluids during the material removal process. Flood coolant is commonly used technique for various machining applications. However, this method uses very large amounts of cutting thus becomes a liability especially on the health and environmental hazards [2]. Therefore, near dry machining was introduced as an alternative to the flood coolant. Reports showed that the performance of near dry machining is superior to that of flood coolant [3-8]. However, in recent days, cryogenic technique was introduced to remove the heat from the cutting zone by using liquid nitrogen as a cooling gas. Besides that, carbon dioxide gas can be used as the cryogenic coolant where the gas needs to be pressurized up to the supercritical phase.

Supercritical carbon dioxide $\left(\mathrm{SCCO}_{2}\right)$ cooling technique is one of the novel techniques that could minimize the generation of cutting temperature and proven to benefit the economical aspect by substituting the aqueous emulsion and the mineral oil, which are currently being used in the metalworking industries. Sprays of rapidly expanding $\mathrm{SCCO}_{2}$ cool and lubricate the machining processes by delivering a mixture of dry ice and lubricant deep into the cutting zone $[9,10]$. From previous researches, $\mathrm{SCCO}_{2}$ cooling technique has shown better results in machining performance in terms of cutting temperature, cutting force, surface quality and tool life compared to minimum quantity lubrication (MQL) and dry machining techniques $[9,10]$. The lubricant flow rate of the continuous $\mathrm{SCCO}_{2}$ spray system used is between 2.41 to $2.61 \mathrm{l} / \mathrm{hr}$ while the mass flow rate of the carbon dioxide $(\mathrm{CO} 2)$ gas is $0.28 \mathrm{~kg} / \mathrm{min}$. Pulse spray mode is seen to be the potential research initiative in reducing the consumption rates that leads to an economic benefit by saving the excessive costs of using metal working fluids. Throughout this work, the ability of $\mathrm{SCCO}_{2}$ with pulse spray mode to produce good surface quality by reducing the generated heat and lubricating the interacting surfaces is investigated. Subsequent reduction of friction and cutting forces are the main focus of this study on the efficiency of the $\mathrm{SCCO}_{2}$ spray pulse during machining. 


\section{EXPERIMENTAL SET UP}

The applicable cutting parameters of the experiment have been considered prior starting the machining operation. The details of machining parameters are shown in TABLE I. . There are three condition of pulse modes used for current work. The cutting speed, feed rate, depth of cut, nozzle distance and nozzle diameter are fixed to be constant throughout the machining experiments. Meanwhile, three levels of input chamber pressure and pulse modes are selected as the machining variables. Fig. 1 shows the machine assembly of the $\mathrm{SCCO}_{2}$ cooling system. AISI 1045 medium carbon steel cylinder with $100 \mathrm{~mm}$ width and $150 \mathrm{~mm}$ length was used as the cutting material. An uncoated cemented carbide tool T1200A with chip breaker and negative rake angle of $14^{\circ}$ was used as the cutting tool during the turning process. The material removal process was conducted on an NC lathe machine (Alpha 400).

The cooling and flow rates of the $\mathrm{SCCO}_{2}$ cryogenic coolant were evaluated prior to the machining process. The results of different cooling and flow rates with input chamber pressure and pulse spray mode dependencies were compared with the continuous spray mode. The lubricant used as a mixture to the cryogenic coolant is synthetic ester and its properties are shown in Table I. The supercritical carbon dioxide exists at a temperature and pressure, which is equal to or greater than the critical temperature and pressure of the $\mathrm{CO}_{2}$ gas which are about $31.2^{\circ} \mathrm{C}$ and $7.38 \mathrm{MPa}$ respectively [11]. At this level, the carbon dioxide molecule has properties between those of a liquid and a gas.

TABLE I. Machining Parameters

\begin{tabular}{l|l}
\hline Cutting parameter & \multicolumn{1}{|c}{ Values } \\
\hline Cutting speed, $v_{c} \quad(\mathrm{~m} / \mathrm{min})$ & 300 \\
Feed rate, $f_{r} \quad(\mathrm{~mm} / \mathrm{rev})$ & 0.2 \\
Depth of cut, $a_{p} \quad(\mathrm{~mm})$ & 1 \\
\hline SCCO $_{2}$ parameter & \multicolumn{1}{|c}{ Values } \\
\hline Input chamber pressure, $P_{c}(\mathrm{MPa})$ & $7.58 ; 8.96 ; 10.4$ \\
Pulse mode (Spray On : Spray Off) & $70: 30,50: 50,30: 70$ \\
Nozzle distance (mm) & 8 \\
Nozzle diameter $(\mathrm{mm})$ & 1.8 \\
\hline Lubricant properties & \multicolumn{1}{|c}{ Values } \\
\hline Type & Synthetic Ester \\
Kinematic viscosity, $v$ at $40^{\circ} \mathrm{C}$ in $\mathrm{mm}^{2} / \mathrm{s}$ & 19 \\
Kinematic viscosity, $v$ at $100^{\circ} \mathrm{C}$ in $\mathrm{mm}^{2} / \mathrm{s}$ & 4.3 \\
Viscosity Index, VI & 137 \\
\hline
\end{tabular}
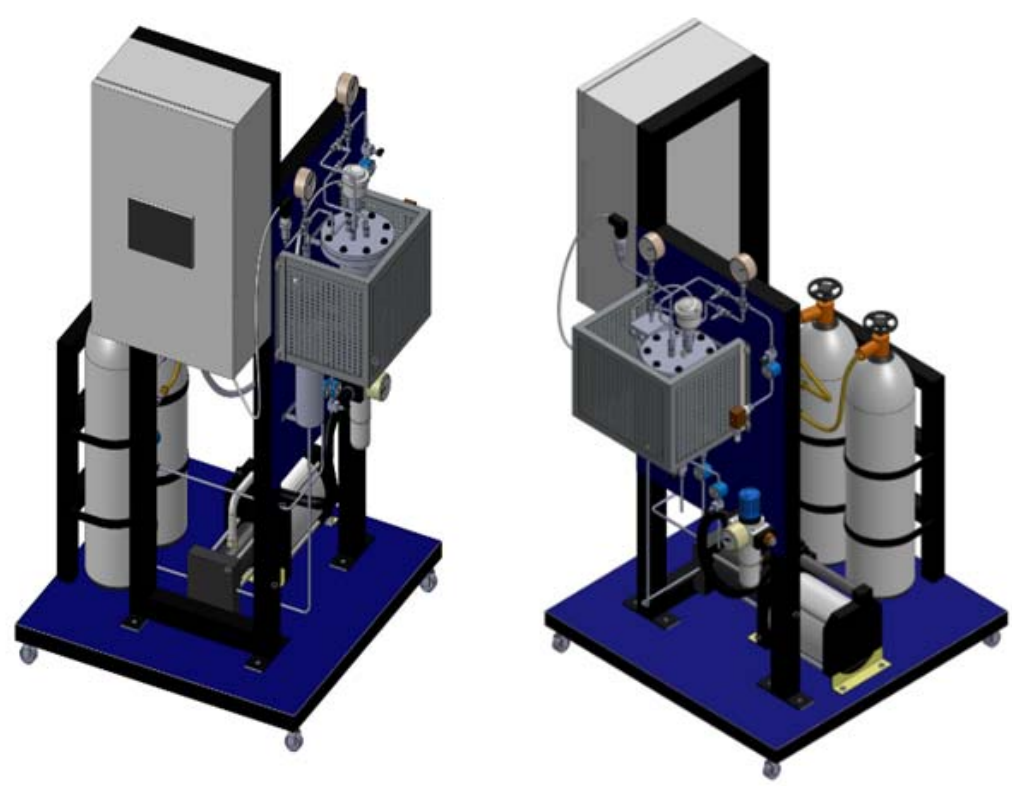

Fig. 1. $\mathrm{SCCO}_{2}$ cooling system (Patent filed) 


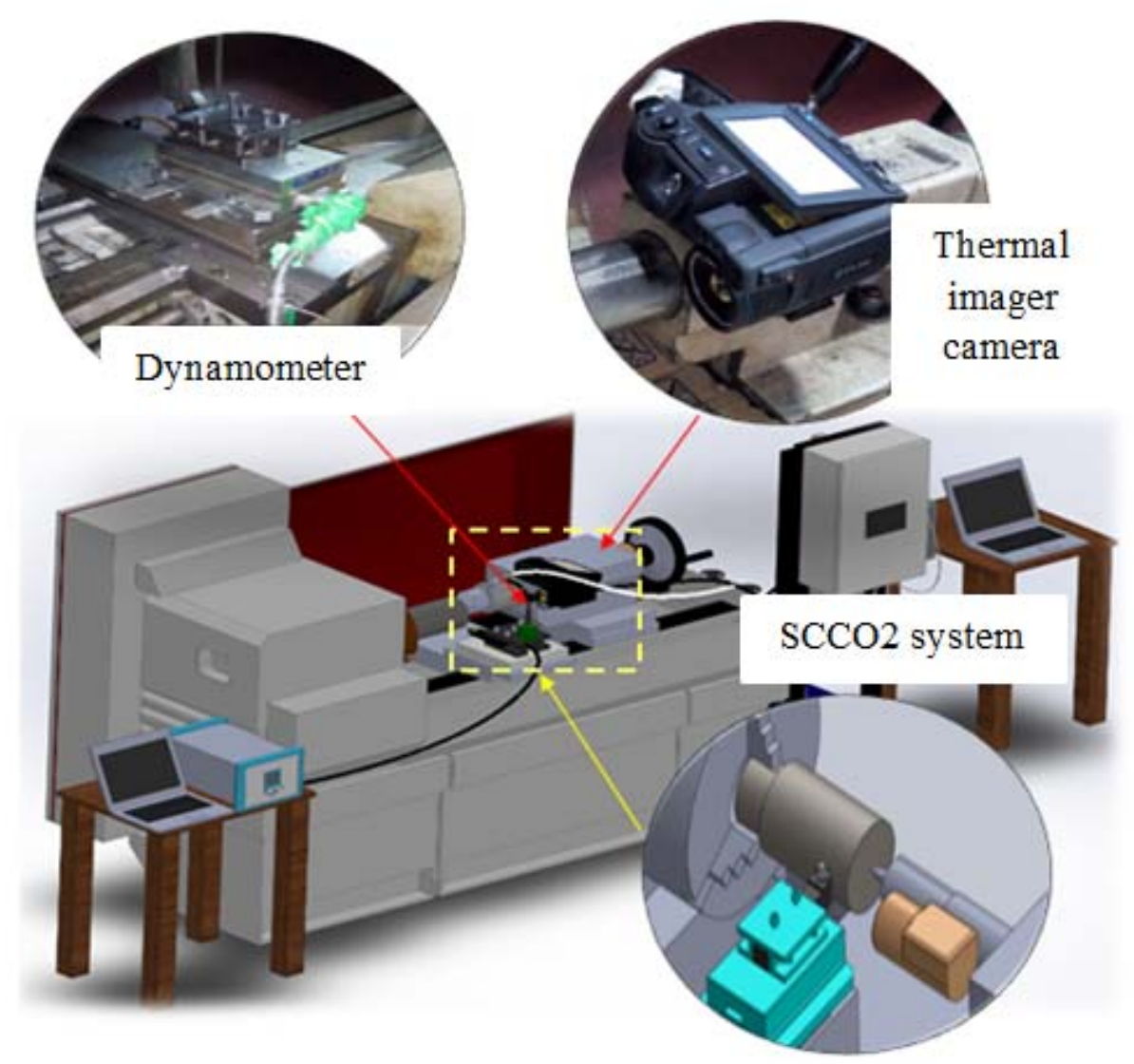

Fig. 2. Machining experimental setup

\section{A. Machining Procedure}

In this study, the value of cutting speed, feed rate and depth of cut were kept constant. Three levels of input chamber pressure, $P_{c}$ and pulse mode were used as the machining parameter variables. In the experiment, three types of pulse mode $(70: 30,50: 50,30: 70)$ were applied. Pulse mode 70:30 means that the lubricant is sprayed for 70 centi-seconds and paused for 30 centi-seconds before the next spray. The time for each spray cycle was 100 centi-seconds.

The data collection of temperature and cutting forces exerted on the tool-workpiece interface was done by using a thermal imager (FLIR) and a dynamometer respectively. As can be seen in Fig. 2, the data acquired by the dynamometer was amplified and processed via a data acquisition card before displaying the results on a computer. After one machining cycle, the workpiece was measured for surface roughness at four different locations by using a portable Mitutoyo surface roughness tester (SJ-400). The cut off distance selected for the surface roughness measurement was $0.8 \mathrm{~mm}$ with a total measurement length of $5.6 \mathrm{~mm}$. The DIN EN ISO 4288 standard is used as a reference for this measurement. The average value of four roughness measurements is then taken for analysis.

\section{III.RESULTS AND DISCUSSION}

In this section, the results from the experimental design and methods used are presented and discussed.

\section{A. Cooling Rate}

It can be seen in Fig. 3, that the value of cooling rate increases when the input chamber pressure increases. The lowest cooling rate is recorded at $0.974{ }^{\circ} \mathrm{C} / \mathrm{s}$ with the input chamber pressure of $7.58 \mathrm{MPa}$ and low pulse mode condition of 30:70. The highest cooling rate which indicates the fastest time to cool the heat on the metal surface off at approximately $8.6^{\circ} \mathrm{C} / \mathrm{s}$ is exhibited by the input chamber pressure of $10.4 \mathrm{MPa}$ with 70:30 pulse mode condition. The lowest value of the cooling rate is caused by the small quantity of cooling medium delivered during the spray. Low quantity of coolant has decreased the spray effectiveness in reducing the material temperature. 


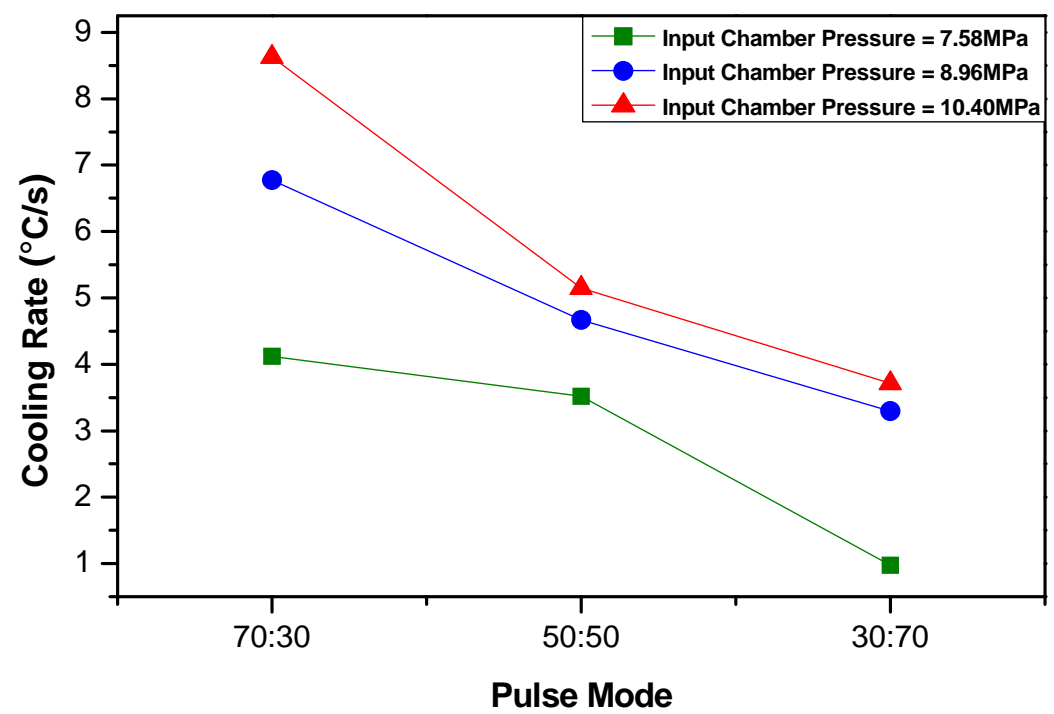

Fig. 3. Cooling rate under different pulse mode and chamber pressure

\section{B. Flow Rate}

Fig. 4 shows the result of $\mathrm{SCCO}_{2}$ flow rate experiment on different pulse spray mode at all three levels of input chamber pressure, Pc and a continuous spray mode as a comparison. At the lowest value of Pc of 7.58 $\mathrm{MPa}$ and pulse mode of 30:70, the smallest quantity of lubricant produced is $1.12 \mathrm{l} / \mathrm{hr}$. The enhancement of input chamber pressure causes the increment of flow rate. This is due to the increase in volumetric flow rate when increasing the pressurized gas through the pressure chamber. Other than that, the pulse mode of 70:30 depicts the longest 'spray on' and shortest 'spray off' condition, thus produces the highest flow rate when compared to the other pulse mode conditions. This effect is also supported by the result depicted by the continuous spray condition whereas it shows the highest value of flow rate compared to others.

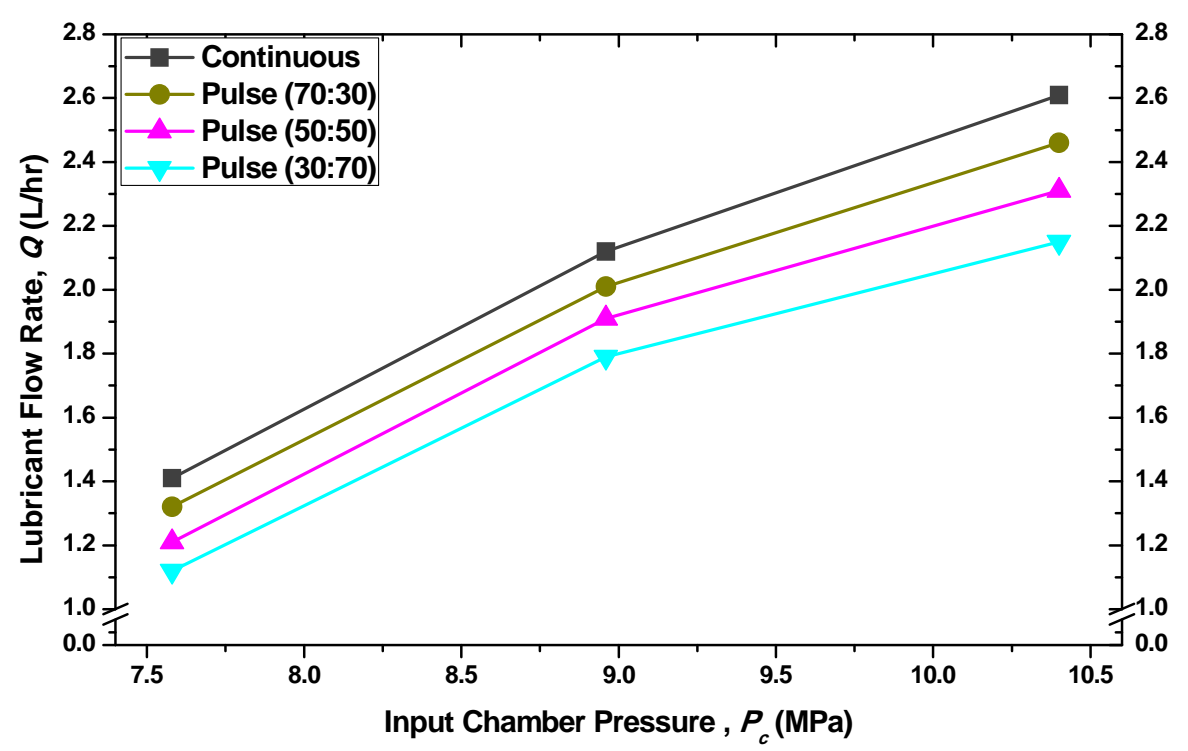

Fig. 4. Flow rate under varied pulse modes and chamber pressure

\section{Cutting Force}

Fig. 5 shows the result of cutting forces under different spray modes and the three levels of $P_{c}(7.58,8.96$ and $10.4 \mathrm{MPa}$ ). When $P_{c}$ is increased, the flow rate of the cutting lubricant is also increased. This causes the amount of cutting fluid that managed to penetrate through the cutting zone to increase. Thus, the friction between the tool surface and workpiece has consequently lowered. As the friction force is smaller, the cutting force is subsequently lessened [12].

On the duration of 'spray on', it is seen that with the shorter time of spray mode, the flow rate also drops. As been discussed before, the amount of cutting fluid that managed to penetrate through the cutting zone provides lubricity to the metal sliding surfaces. By further lowering the lubricity effect on the tool-workpiece contacts, 
the cutting performance shows a declining trend. The different condition of pulse mode resulted in the difference of spray duration. The trend of increasing cutting force may be explained by the shorter time of 'spray on' that subsequently decreases the quantity of lubricant flow and cooling rate delivered to the cutting surface.

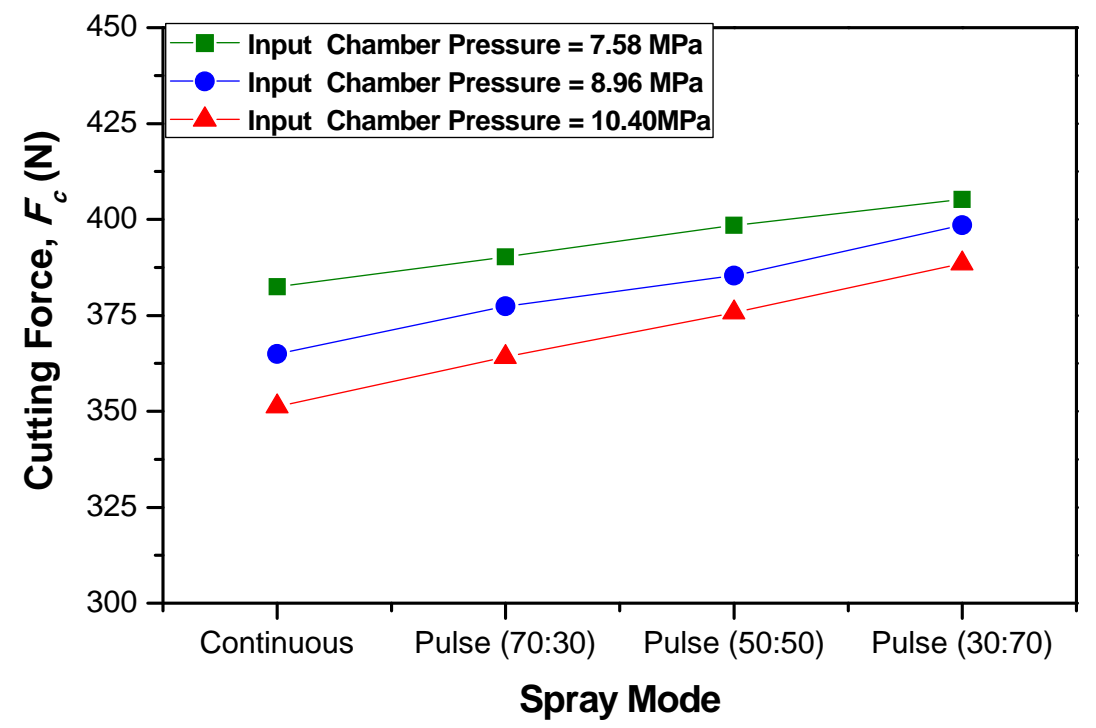

Fig. 5. Cutting force under different pulse modes and chamber pressure $\left(P_{c}\right)$

\section{Cutting Temperature}

Cutting temperature measured from each machining parameters are shown in Fig. 6. The spray pulse mode of 70:30 produces the highest quantity of flow rate than the other spray conditions. The longer the 'spray on' mode, the longer is the flow rate of the cutting lubricant. Adequate amount of lubricant mixed in the cryogenic cooling medium on the machining surfaces reduces the mechanical damage between the tool surface and the workpiece. This explained the high cooling capacity of the cutting fluid that lubricates the cutting zone. The high volume of flow rate lowers the cutting temperature by improving the convection and heat transfer rate [12]. With the high cooling capacity of the $\mathrm{SCCO}_{2}$ medium, it is observed that during the material removal process, the temperature is slightly increased with the decrement of $P_{c}$.

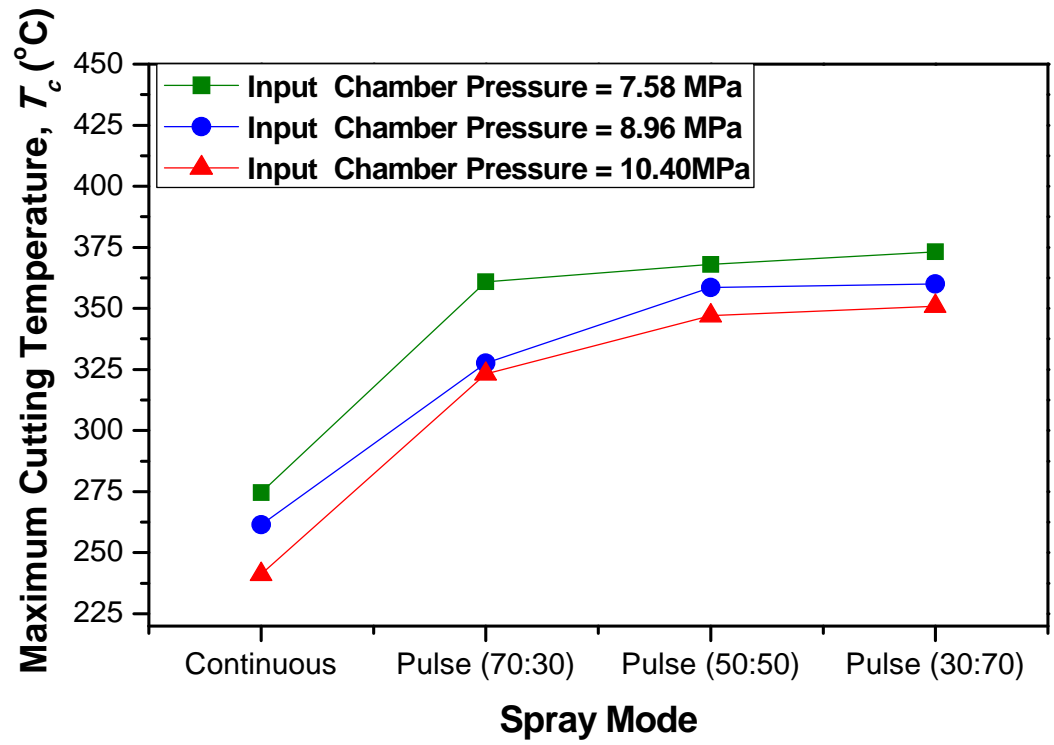

Fig. 6. Cutting temperature under different pulse modes and chamber pressure $\left(P_{c}\right)$

The results from flow rate and cooling rate experiments (Figs. 3 and 5) are also correlated to the results depicted in Fig. 6. The high input chamber pressure that produced high flow and cooling rates causes the increase of lubricant quantity on the cutting zone. The natural convection of cryogenic coolant delivered on the metal surface leads to the formation of frost which decreases the temperature of the contacting surfaces. The mixture of the cryogenic gas with lubricant concurrently improved the anti-friction and the lubricating effect of 
the cutting fluids. The high energy dissipation at the cutting zone strongly helps in lowering the generated heat $[12,13]$.

\section{E. Surface Roughness}

Fig. 7 shows the influence of $P_{c}$ and the cooling spray mode on the arithmetic average of the roughness profile, $R_{a}$ on the machined surface. At the continuous spray mode, the roughness profile is almost constant independent of the cooling spray mode settings. This effect is common for turning as the feed rate was set to be constant for all experiments [14]. It is also shown that the roughness values in continuous spray modes are lower than its counterparts with the lowest is observed at the highest $P_{c}$. On the other hand, the shortest 'spray on' duration depicts the highest surface quality also at the highest $P_{c}$, comparable to the continuous spray mode. Complete lubrication of the cutting zone by the application of continuous cryogenic coolant mixture flow with high input chamber pressure may explain the effect of the reducing $R_{a}$ value. The induced hardening effect of the workpiece by the cryogen helps in forming brittle uncut surface which enhances chip breakability, decreasing the cutting force and temperature (cf. Figs. $5 \& 6$ ) and thus, improving the surface quality [15].

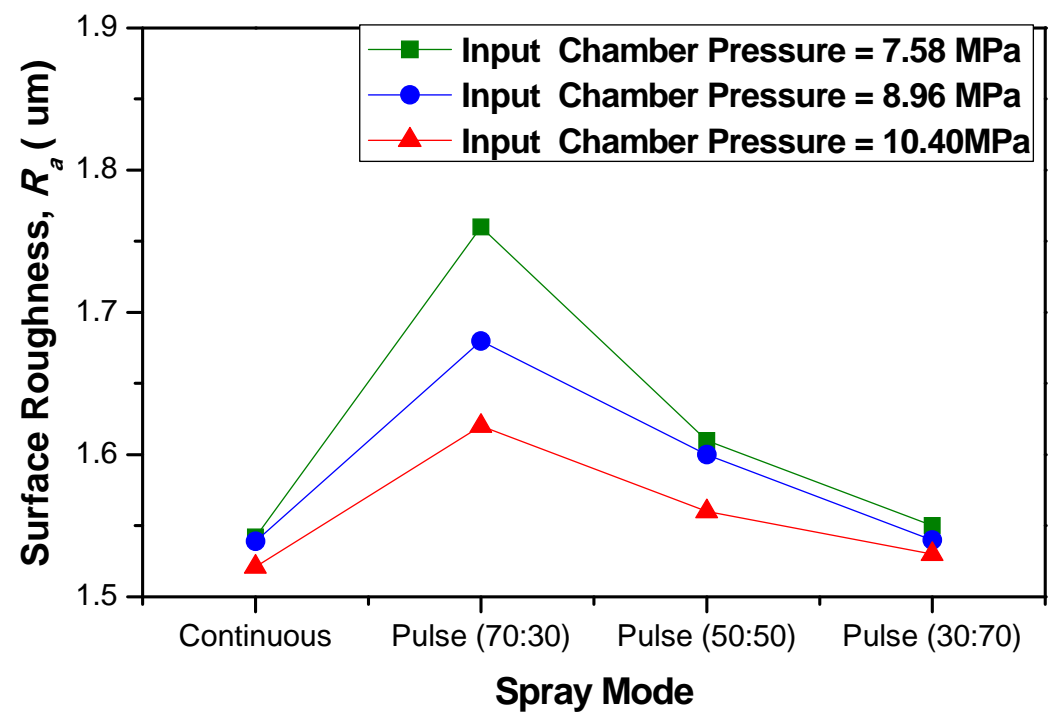

Fig. 7. Surface roughness temperature under different pulse modes and chamber pressure $\left(P_{c}\right)$

The same explanation is also observed during machining with the lowest 'spray on' duration of 30:70, whereby a nearly dry machined surface was introduced on the machining surface, which subsequently increases the workpiece temperature close to the cutting zone (cf. Fig. 6). The high temperature decreases the strength and hardness of the uncut surface layer that eases the chip formation which subsequently improve the surface quality [16]. In addition, with increasing $P_{c}$, roughness decreases marginally dependent on the volumetric flow rate of the coolant. The high pressurised gas sprayed towards the cutting zone also plays a secondary function by avoiding chip entanglements at the cutting tool from damaging the machined surface. Nevertheless, all surface qualities depicted in Fig. 7 are within the acceptable range of good surface finish below than $2 \mu \mathrm{m}$.

\section{F. Tool Life}

Tool life increases with the increase of both input chamber pressure and 'spray on' duration as shown in Fig. 8. Maximum tool life at the cutting distance of $26650 \mathrm{~mm}$ and 133.25 minutes of machining time are obtained at high input chamber pressure of 10.4 MPa when using continuous spray technique. Meanwhile, the minimum tool life of 41 minutes is observed at the lowest input chamber pressure and pulse spray duration of $7.58 \mathrm{MPa}$ and pulse mode of 30:70 respectively. A cutting distance at only $8200 \mathrm{~mm}$ is observed at this spray mode condition. It shows that a rapid tool wear is occurred after only a short period of cutting time. These results are related to the cutting force and cutting temperature. Lower cutting force and temperature obtained during the turning operations are the results of better lubricant flow and cooling rate at the cutting zone. The quantity of lubricants increases and the lubricity effect of the cryogenic coolants in the cutting zone is turned out to be very high. The higher quantity of lubricant helps in reducing the friction and decreasing the cutting temperature and subsequently lowering the tool wear rate as well as extending the tool life. 

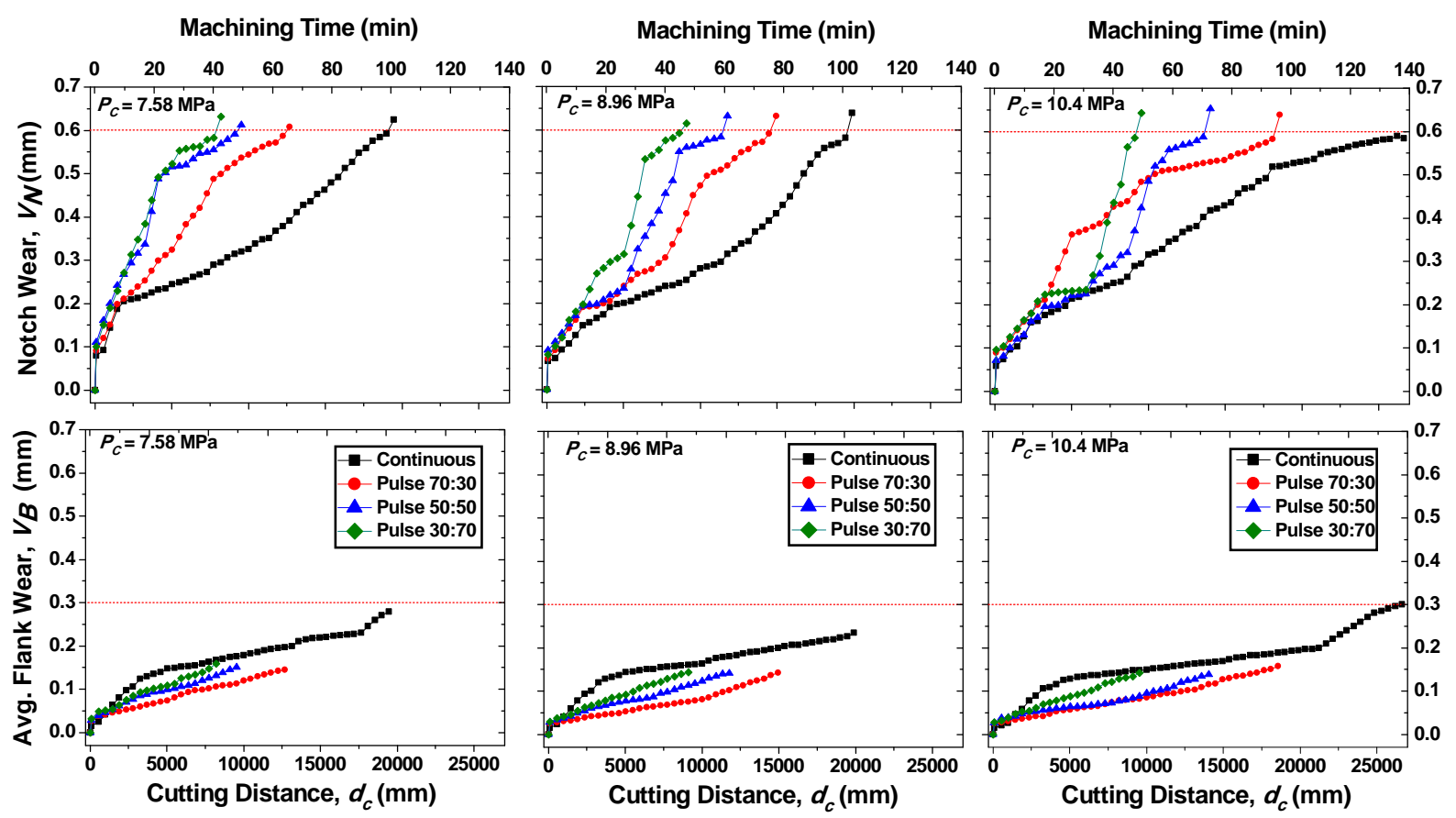

Fig. 8. Tool life under different pulse modes and chamber pressure $\left(P_{c}\right)$

It can be observed that notch wear is the dominant tool wear mode. One of the independent effects is the stress distribution at the tool-chip interfaces, which is influenced by the effective tool-chip contact area and the contact mechanics. The increased of chip thickness, friction and cutting force was affected by the increase of contact length [17]. The increased friction between tool-chip interfaces causes more tearing and rubbing with the newly generated chips and the rubbed parts of the chips stick or are essentially friction welded to the tool [18]

\section{IV.CONCLUSIONS}

At the end of this study, the effectiveness of $\mathrm{SCCO}_{2}$ cooling technique with different pulse mode was successfully evaluated. From the value of cutting temperature, cutting force and surface roughness, these conclusions were obtained.

i. The application of pulse mode in supercritical carbon dioxide $\left(\mathrm{SCCO}_{2}\right)$ cooling technique is suggested as a good solution for cutting lubricant consumption reduction. The long "spray on" mode of 70:30 with the highest input chamber pressure depicts good comparable results to the continuous spray mode especially in reducing the cutting force, temperature and improving the tool life due to their good volumetric flow rate and cooling effect.

ii. The surface quality obtained after the cutting processes with continuous spray mode indicates the lowest roughness value and comparable to the nearly dry machining technique shown by the shortest "spray on" mode of 30:70. However, all the other surface qualities are within the acceptable range of good surface finish below than $2 \mu \mathrm{m}$.

iii. The highest input chamber pressure of $10.4 \mathrm{MPa}$ is shown to play a very important role in combination with the pulse spray modes that reduces the friction coefficient and cutting forces, which subsequently improves the tool life.

iv. The performance of $\mathrm{SCCO}_{2}$ pulse cooling is therefore comparable with continuous cooling with only $3.64 \%$ of cutting force increment, $34 \%$ of temperature increment, $14 \%$ of surface roughness increment and $36.18 \%$ of tool life increment.

\section{ACKNOWLEDGMENT}

The authors thank the Ministry of Education Malaysia and Universiti Tun Hussein Onn Malaysia for the financial support via FRGS (Vot 1218). 


\section{REFERENCES}

[1] D. A. Stephenson and S. A. John. Metal Cutting Theory and Practice, 2nd ed., USA: Taylor and France; 2006.

[2] E. A. Rahim and H. Sasahara. "An analysis of surface integrity when drilling inconel 718 using palm oil and synthetic ester under MQL condition,” Mach. Sci. Technol., vol. 15(1), pp. 76-90, Mar. 2011.

[3] E. A. Rahim and H. Sasahara. "Application of minimum quantity lubrication when drilling nickel-based superalloy at high cutting speed,” Key Eng. Mat., vol. 407 - 408, pp. 612-615, Feb. 2009.

[4] E. A. Rahim and H. Sasahara. "Effect of MQL liquids on surface integrity when high speed drilling titanium alloy," Key Eng. Mat., vol. 443, pp. 359-364, June 2010.

[5] E. A. Rahim and H. Sasahara. "Surface integrity when drilling nickel-based superalloy under MQL supply," Key Eng. Mat., vol. 443, pp. 365-370, June 2010.

[6] E. A. Rahim and H. Sasahara. "Surface integrity in MQL drilling nickel-based superalloy," Key Eng. Mat., vol. 447 - 448, pp. 811815, Sept. 2010.

[7] E. A. Rahim and H. Sasahara. "Effect of machining parameters and MQL liquids on surface integrity of high speed drilling Ti-6Al4V," Key Eng. Mat., vol. 447-448, pp. 816-820, Sept. 2010.

[8] E. A. Rahim and H. Sasahara. "Investigation of tool wear and surface integrity on MQL machining of Ti-6Al-4V using biodegradable oil,” Proc. Inst. Mech. Eng. Part B J. Eng. Manuf., vol. 225(9), pp. 1505-1511, Feb. 2011.

[9] S. D. Supekar, A. F. Clarens, D. A. Stephenson and S. J. Skerlos. "Performance of supercritical carbon dioxide sprays as coolants and lubricants in representative metalworking operations," J. Mater. Process. Technol., vol. 212, pp. 2652-2658, Dec. 2012.

[10] E. A. Rahim, A. A. Rahim, M. R. Ibrahim and Z. Mohid. "Experimental Investigation of Supercritical Carbon Dioxide $\left(\mathrm{SCCO}_{2}\right)$ Performance as a Sustainable Cooling Technique," Procedia CIRP, vol. 40, pp. 638-642, Feb. 2016.

[11] J. W. Tom, and P. G. Debenedetti. "Particle formation with supercritical fluids-a review," Journal of Aerosol Science, vol. 22, pp. 555-584, Dec. 1990.

[12] E. A. Rahim, A. A. Rahim, Z. Mohid and A.S.A. Sani. "Effects of Supercritical Carbon Dioxide $\left(\mathrm{SCCO}_{2}\right)$ Cooling Technique on the Evolution of Machining Performance," in Proc. of LEM21, 2015, Kyoto, Japan, paper 0208.

[13] E. G. Fryderyk. Application of Metal Cutting Theory. New York: Industrial Press Inc.; 1987.

[14] J. C. Aurich, P. Mayer, B. Kirsch, D. Eiffler, M. Smaga and R. Skorupski. "Characterization of deformation induced surface hardening during cryogenic turning of AISI 347," CIRP Annals - Manuf. Technol., vol. 63, pp. 65-68, Apr. 2014.

[15] Z. Zhao, and S. Y. Hong. "Cooling strategies for cryogenic machining from a materials viewpoint," Journal of Materials Engineering and Performance, vol. 1, pp. 669-678, Oct. 1992.

[16] A. E. Diniz and R. Micaroni. "Cutting conditions for finish turning process aiming: the use of dry cutting," International Journal of Machine Tools \& Manufacture, vol. 42, pp. 899-904, March 2002.

[17] P. L. B. Oxley. "An analysis for orthogonal cutting with restricted tool-chip contact,” Int. J. Mech. Sci., vol. 4, pp. 129-135, Mar.-Apr. 1962.

[18] Y. Kaynak, H. E. Karaca, R. D. Noebe and I. S. Jawahir. "Tool-wear analysis in cryogenic machining of NiTi shape memory alloys: A comparison of tool-wear performance with dry and MQL machining," Wear, vol. 306, pp. 51-63, Aug. 2013.

\section{AUTHOR PROFILE}

Erween Abd Rahim (Rahim E. A.) is an Associate Professor at the Universiti Teknologi Tun Hussein Onn Malaysia and the Head of the Precision Machining Research Centre at the Department of Manufacturing and Industrial Engineering, Faculty of Mechanical and Manufacturing Engineering, UTHM. 